M. Shirosaki

Nagoya Math. J.

Vol. 120 (1990), 103-112

\title{
ON DEFECT RELATIONS OF MOVING HYPERPLANES
}

\author{
MANABU SHIROSAKI
}

\section{§1. Introduction}

The defect relation $\sum_{j=1}^{q} \delta\left(f, H_{j}\right) \leq n+1$ gives the best-possible estimate, where $f$ is a linearly non-degenerate holomorphic curve in $P^{n}(C)$ and $H_{1}, \cdots, H_{q}$ are hyperplanes in $P^{n}(C)$ which are in general position. However, the case of moving hyperplanes has ever got only $n(n+1)$ instead of $n+1$ (Stoll [4]) and it has not yet been known whether this bound is best-possible or not. In this paper we shall give some particular cases which have the bound $n+1$.

The author thanks Professor Fujimoto for his useful advice and discussions with the author.

\section{§2. Holomorphic curves and moving hyperplanes}

In this paper, we fix one homogeneous coordinate system of the $n$ dimensional complex projective space $P^{n}(C)$ and denote it by the notation $w=\left(w_{0}: \cdots: w_{n}\right)$.

A hyperplane $H$ in $P^{n}(C)$ is an $(n-1)$-dimensional projective subspace of $P^{n}(\boldsymbol{C})$, i.e., it is given by $H=\left\{w \in P^{n}(C) \mid \sum_{j=0}^{n} a_{j} w_{j}=0\right\}$, where $\left(a_{0}, \cdots, a_{n}\right) \in C^{n+1}-\{0\}$. We call the vector $\left(a_{0}, \cdots, a_{n}\right)$ a representation of $H$. Let $H_{j}$ be hyperplanes in $P^{n}(C)$ with representations $a^{j}=$ $\left(a_{0}^{j}, \cdots, a_{n}^{j}\right) \quad(j=1, \cdots, q)$. If any $\min (q, n+1)$ elements of $a^{1}, \cdots, a^{q}$ are linearly independent over $C, H_{1}, \cdots, H_{q}$ are said to be in general position.

We call a holomorphic mapping $f: C \rightarrow P^{n}(C)$ a holomorphic curve in $P^{n}(C)$. A representation of $f$ is a holomorphic mapping $\tilde{f}=\left(f_{0}, \cdots, f_{n}\right)$ : $C \rightarrow C^{n+1}$ which satisfies $\tilde{f}^{-1}(0) \neq C$ and $f(z)=\left(f_{0}(z): \cdots: f_{n}(z)\right)$ for all $z \in$ $C-\tilde{f}^{-1}(0)$. Then we write $f=\left(f_{0}: \cdots: f_{n}\right)$. If $\tilde{f}^{-1}(0)=\varnothing$, then the representation $\tilde{f}$ is said to be reduced.

Definition 2.1. A moving hyperplane $H^{M}$ in $P^{n}(C)$ is a mapping of

Received December 15, 1989. 
$C$ into the set of all hyperplanes in $P^{n}(C)$ given by $H^{M}(z)=\left\{w \in P^{n}(C) \mid\right.$ $\left.\sum_{j=0}^{n} a_{j}(z) w_{j}=0\right\}(z \in C)$, where $\left(a_{0}, \cdots, a_{n}\right)$ is a reduced representation of some holomorphic curve $g$ in $P^{n}(C)$. We call a representation and a reduced representation of $g$ a representation and a reduced representation of $H^{M}$, respectively.

Definition 2.2. Let $H_{j}^{M}$ be moving hyperplanes in $P^{n}(C) \quad(j=1$, $\cdots, q) . \quad H_{1}^{M}, \cdots, H_{q}^{M}$ are said to be in general position if there exists a point $z_{0}$ of $C$ such that hyperplanes $H_{1}^{M}\left(z_{0}\right), \cdots, H_{q}^{M}\left(z_{0}\right)$ in $P^{n}(C)$ are in general position.

Definition 2.3. Let $f$ be a holomorphic curve in $P^{n}(C)$ with a representation $\left(f_{0}, \cdots, f_{n}\right)$ and let $K$ be an extension field of $C$. We say that $f$ is non-degenerate over $K$ if $f_{0}, \cdots, f_{n}$ are linearly independent over $K$. In particular, $f$ is said to be linearly non-degenerate if it is nondegenerate over $\boldsymbol{C}$.

\section{$\S 3$. Characteristic functions, counting functions and defects}

We define the norm $\|\boldsymbol{z}\|$ of $\boldsymbol{z}=\left(\boldsymbol{z}_{1}, \cdots, z_{m}\right) \in \boldsymbol{C}^{m}$ by $\|\boldsymbol{z}\|^{2}=\sum_{j=1}^{m}\left|\boldsymbol{z}_{j}\right|^{2}$.

DeFinition 3.1. The characteristic function of a holomorphic curve $f$ in $P^{n}(C)$ with a reduced representation $\tilde{f}$ is defined for $0<s<r$ by

$$
T(f ; r, s)=\frac{i}{2 \pi} \int_{s}^{r} \frac{d t}{t} \int_{|z| \leq t} \partial \overrightarrow{\hat{o}} \log \|\tilde{f}\|^{2} .
$$

This definition does not depend on the choice of $\tilde{f}$. We see that $T(f ; r, s)$ is non-negative and that if $f$ is non-constants, then $T(f ; r, s)$ $\rightarrow \infty$ monotonically as $r \rightarrow \infty$. Furthermore we can easily verify that

$$
T(f ; r, s)=\frac{1}{2 \pi} \int_{0}^{2 \pi} \log \left\|\tilde{f}\left(r e^{i \theta}\right)\right\| d \theta-\frac{1}{2 \pi} \int_{0}^{2 \pi} \log \left\|\tilde{f}\left(s e^{i \theta}\right)\right\| d \theta .
$$

Definition 3.3. The counting function of zeros for a meromorphic function $F \not \equiv 0$ on $C$ is defined for $0<s<r$ by

$$
N(F ; r, s)=\int_{s}^{r} n(F ; t) \frac{d t}{t},
$$

where $n(F ; t)$ is the sum of zero orders of $F$ in $\{\boldsymbol{z} \in \boldsymbol{C}|| z \mid \leq t\}$.

By the definition, $N(F ; r, s)$ is non-negative, and Jensen's formula shows that 


$$
\begin{aligned}
& N(F ; r, s)-N(1 / F ; r, s) \\
& \quad=\frac{1}{2 \pi} \int_{0}^{2 \pi} \log \left|F\left(r e^{i \theta}\right)\right| d \theta-\frac{1}{2 \pi} \int_{0}^{2 \pi} \log \left|F\left(s e^{i \theta}\right)\right| d \theta .
\end{aligned}
$$

In the situation of Definition 2.1, we define the characteristic function of $H^{M}$ by $T\left(H^{M} ; r, s\right):=T(g ; r, s)$. And we define the counting function of $H^{M}$ for a holomorphic curve $f$ by $N\left(f, H^{M} ; r, s\right):=N((\tilde{f}, \tilde{\mathbf{g}}) ; r, s)$, where $\tilde{f}=\left(f_{0}, \cdots, f_{n}\right)$ and $\tilde{g}=\left(a_{0}, \cdots, a_{n}\right)$ are reduced representations of $f$ and $g$, respectively, and $(\tilde{f}, \tilde{g}):=\sum_{j=0}^{n} a_{j} f_{j}$, if $(\tilde{f}, \tilde{g}) \neq \equiv 0$. This assumption holds if $f$ is non-degenerate over a field containing all $a_{f} / a_{k}$ with $a_{k} \neq \equiv 0$. This definition does not depend on the choice of $\tilde{f}$ and $\tilde{g}$. By (3.2), (3.4) and Schwarz's inequality, we get

$$
N\left(f, H^{M} ; r, s\right) \leq T(f ; r, s)+T\left(H^{M} ; r, s\right)+O(1), \quad r \longrightarrow \infty .
$$

If either $f$ or $g$ is not constant, the defect of $H^{M}$ for $f$ is defined by

$$
\delta\left(f, H^{M}\right)=\liminf _{r \rightarrow \infty}\left(1-\frac{N\left(f, H^{M} ; r, s\right)}{T(f ; r, s)+T\left(H^{M} ; r, s\right)}\right)
$$

which does not depend on $s$. By (3.5), we see $0 \leq \delta\left(f, H^{M}\right) \leq 1$. The moving hyperplane $H^{M}$ is said to be of lower order than $f$ if $T\left(H^{M} ; r, s\right)$ $=o(T(f ; r, s))$ as $r \rightarrow \infty$. Then

$$
\delta\left(f, H^{M}\right)=\liminf _{r \rightarrow \infty}\left(1-\frac{N\left(f, H^{M} ; r, s\right)}{T(f ; r, s)}\right) .
$$

The definitions of counting functions and defects of (not-moving) hyperplanes are the same as those of moving hyperplanes. However, for convenience sake, we consider that the category of moving hyperplanes contains not-moving hyperplanes.

Let $f$ be a holomorphic curve in $P^{n}(C)$. We denote by $K_{f}$ the set of all meromorphic functions $g$ which satisfy the condition that $T(g ; r, s)$ $=o(T(f ; r, s))$ as $r \rightarrow \infty$. If a representation $\left(f_{0}, \cdots, f_{n}\right)$ satisfies that $f \not \equiv 0$ for each $j$ and that each $f_{j} / f_{k}(j \neq k)$ is not constant, then we set $\tilde{K}_{f}=\cap_{j \neq k} K_{f_{j} / f_{k}}$. Now, we present two lemmas without proofs.

LEMma 3.6 ([4, Lemma 5.3]). The sets $K_{f}$ and $\tilde{K}_{f}$ are fields.

Lemma 3.7 ([1, Proposition 5.9]). A holomorphic curve $f=\left(f_{0}: \cdots: f_{n}\right)$ in $P^{n}(C)$ is rational, i.e., all $f_{j} \mid f_{k}$ with $f_{k} \neq \equiv$ are rational if and only if

$$
T(f ; r, s)=O(\log r) \quad \text { as } r \longrightarrow \infty \text {. }
$$


Proposition 3.8. Let $f$ be a non-constant holomorphic curve and let $g$ be a holomorphic curve in $P^{n}(C)$ with a reduced representation ( $g_{0}$, $\left.\cdots, g_{n}\right)$. Assume that $g_{j} / g_{k} \in K_{f}$ if $g_{k} \not \equiv 0$. Then, $T(g ; r, s)=o(T(f ; r, s))$ as $r \rightarrow \infty$.

Proof. Without loss of generality, we may assume that $g_{0} \not \equiv 0$. Since the representation $\left(g_{0}, \cdots, g_{n}\right)$ is reduced, for each point $p$ where $g_{0}$ vanishes there is some $g_{j}$ with $g_{j}(p) \neq 0$. Hence, we have

$$
\begin{aligned}
N\left(g_{0} ; r, s\right) & \leq \sum_{j=1}^{n} N\left(g_{j} / g_{0}, \infty ; r, s\right) \\
& \leq \sum_{j=1}^{n} T\left(g_{j} / g_{0} ; r, s\right)+O(1) \\
& =o(T(f ; r, s)) \quad(r \longrightarrow \infty)
\end{aligned}
$$

and

$$
\begin{aligned}
T(g ; r, s)= & \frac{1}{4 \pi} \int_{0}^{2 \pi} \log \left(1+\sum_{\jmath=1}^{n}\left|g_{j}\left(r e^{i \theta}\right) / g_{0}\left(r e^{i \theta}\right)\right|^{2}\right) d \theta \\
& +\frac{1}{2 \pi} \int_{0}^{2 \pi} \log \left|g_{0}\left(r e^{i \theta}\right)\right| d \theta+O(1) \\
\leq & \sum_{j=1}^{n} T\left(g_{j} / g_{0} ; r, s\right)+N(g ; r, s)+O(1) \\
= & o(T(f ; r, s)) \quad(r \longrightarrow \infty) .
\end{aligned}
$$

In this paper, we treat non-rational holomorphic curves $f$ and we use a notation $S(f, r)$ for representing a quantity with a property that

$$
\lim _{r \rightarrow \infty, r \notin E} S(f ; r) / T(f ; r, s)=0
$$

for a set $E \subset(0, \infty)$ of finite Lebesgue measure.

\section{§4. Defect relations}

First, we give the known defect relations.

Theorem 4.1 (See, for example, [3, Chapter 3]). Let $f$ be a linearly non-degenerate holomorphic curve in $P^{n}(C)$ and let $H_{1}, \cdots, H_{q}$ be hyperplanes in $P^{n}(C)$ which are in general position. Then

$$
\sum_{j=1}^{q} \delta\left(f, H_{j}\right) \leq n+1 .
$$

THEOREM 4.2 ([4, Theorem 6.19]). Let $f$ be a holomorphic curve in $P^{n}(C)$ and let $H_{1}^{M}, \cdots, H_{q}^{M}$ be moving hyperplanes in $P^{n}(C)$ with lower orders than $f$ which are in general position. Let $\left(a_{0}^{j}, \cdots, a_{n}^{j}\right)$ be reduced representations of $H_{j}^{M}(j=1, \cdots, q)$ and $K$ be the smallest extension field 
of $C$ which contains all $a_{k}^{j} / a_{m}^{j}\left(1 \leq j \leq q, 0 \leq k \leq n\right.$, and $\left.m \in\left\{k \mid a_{k}^{j} \neq \equiv 0\right\}\right)$. Assume that $f$ is non-degenerate over $K$. Then

$$
\sum_{j=1}^{q} \delta\left(f, H_{j}^{M}\right) \leq n(n+1) .
$$

Theorem 4.3 (2, Theorem 3.4]). Let $f$ be a holomorphic curve in $P^{n}(\boldsymbol{C})$ and let $H_{0}^{M}, \cdots, H_{n+1}^{M}$ are moving hyperplanes in $P^{n}(C)$ with lower orders than $f$ which are in general position. Let $\left(a_{0}^{j}, \cdots, a_{n}^{j}\right)$ be reduced representations of $H_{j}^{M}(j=0, \cdots, n+1)$ and let $K$ be the smallest extension field of $C$ which contains all $a_{k}^{j} / a_{m}^{j}\left(0 \leq j, k \leq n\right.$ and $\left.m \in\left\{k \mid a_{k}^{j} \not \equiv 0\right\}\right)$. Assume that $f$ is non-degenerate over $K$. Then

$$
\sum_{j=0}^{n+1} \delta\left(f, H_{j}^{M}\right) \leq n+1 .
$$

The main purpose of this paper is to prove the following:

THEOREM 4.4. Let $f$ be a linearly non-degenerate holomorphic curve in $P^{n}(C)$ with a reduced representation $\left(f_{0}, \cdots, f_{n}\right)$ and let $H_{1}^{M}, \cdots, H_{q}^{M}$ be moving hyperplanes in general position in $P^{n}(C)$. Let $\left(a_{0}^{j}, \cdots, a_{n}^{j}\right)$ be reduced representations of $H_{j}^{M}(1 \leq j \leq q)$. Assume that the following three conditions are satisfied:

(C1) $a_{k}^{j} / a_{m}^{j} \in \tilde{K}_{f}$ if $a_{m}^{j} \not \equiv 0$;

(C2) $f$ is non-degenerate over $\tilde{K}_{f}$;

(C3) $N\left(f_{f} ; r, s\right)=S(f ; r) \quad(j=0, \cdots, n)$.

Then

$$
\sum_{j=1}^{q} \delta\left(f, H_{j}^{M}\right) \leq n+1
$$

\section{§5. Second main theorems}

The next second main theorem is well-known and Theorem 4.1 is its corollary:

Theorem 5.1 (See, for example, [3, Chapter 3]). In the same situation of Theorem 4.1, the inequality

$$
(q-n-1) T(f ; r, s) \leq \sum_{j=1}^{q} N\left(f, H_{j} ; r, s\right)+S(f ; r)
$$

holds for $0<s<r$.

The next lemma will be proved by the same method of Theorem 4.3. For a proof, see [5, pp. 313-333]. 
LEMma 5.3. In the same situation of Theorem 4.3, the inequality

$$
T(f ; r, s) \leq \sum_{j=0}^{n+1} N\left(f, H_{j}^{M} ; r, s\right)+S(f ; r)
$$

holds for $0<s<r$.

\section{§6. Proof of Theorem 4.4}

Before begining to prove Theorem 4.4, we show the following lemma.

Lemma 6.1. Let $f$ be as in Theorem 4.4. Let $H^{M}$ be a moving hyperplane in $P^{n}(C)$ with a reduced representation $\left(a_{0}, \cdots, a_{n}\right)$. Assume that $a_{j} / a_{k} \in \tilde{K}_{f}$ if $a_{k} \neq \equiv$. If $a_{j_{0}} \neq \equiv 0, \cdots, a_{j_{k}} \neq 0$ and $a_{j} \equiv 0$ for $j \neq j_{0}, \cdots, j_{k}$, we give a hyperplane $H=\left\{w \in P^{n}(\boldsymbol{C}) \mid w_{j_{0}}+\cdots+w_{j_{k}}=0\right\}$ in $P^{n}(\boldsymbol{C})$. Then

$$
N(f, H ; r, s)=N\left(f, H^{M} ; r, s\right)+S(f ; r) .
$$

Proof. For simplicity, we may assume that $j_{0}=0, \cdots, j_{k}=k$. In the case of $k=0$, the conclusion is evident since $N\left(f_{0} ; r, s\right)=o(T(f ; r, s))$ $(r \rightarrow \infty)$ by Proposition 3.8. Hence we assume that $k \geq 1$.

Let $h:=\left(f_{0}: \cdots: f_{k}\right)$ be a holomorphic curve in $P^{k}(C)$ and let $L^{M}$ be a moving hyperplane in $P^{k}(C)$ with a reduced representation $\left(a_{0}, \cdots, a_{k}\right)$. Furthermore, we consider the hyperplanes $L_{j}:=\left\{w \in P^{k}(C) \mid w_{j}=0\right\} \quad(j=$ $0, \cdots, k)$ and $L:=\left\{w \in P^{k}(\boldsymbol{C}) \mid \sum_{j=0}^{k} w_{j}=0\right\}$ in $P^{k}(\boldsymbol{C})$. Note that $L^{M}$ has a lower order than $h$. We get by Theorem 5.1 and Lemma 5.3.

$$
T(h ; r, s) \leq N\left(h, L^{M} ; r, s\right)+S(f ; r)
$$

and

$$
T(h ; r, s) \leq N(h, L ; r, s)+S(f ; r) .
$$

Here we used the fact $N\left(h, L_{j} ; r, s\right)=S(f ; r)(j=0, \cdots, k)$. By (3.5) and the above inequalities, we have $T(h ; r, s)=N(h, L ; r, s)+S(f ; r)$ and $T(h ; r, s)=N\left(h, L^{M} ; r, s\right)+S(f ; r)$. Since $N(h, L ; r, s)+o(T(f ; r, s))=$ $N(f, H ; r, s)$ and $N\left(h, L^{H} ; r, s\right)=N\left(f, H^{H} ; r, s\right)+o(T(f ; r, s))(r \rightarrow \infty)$, we obtain (6.2).

Q.E.D.

Proof of Theorem 4.4. There exists a point $z_{0}$ of $C$ such that $a_{k}^{j}\left(z_{0}\right)$ $\neq 0$ if $a_{k}^{j} \not \equiv 0$ and that $H_{1}^{M}\left(z_{0}\right), \cdots, H_{q}^{M}\left(z_{0}\right)$ are in general positions. Then by Lemma 6.1, we have

$$
N\left(f, H_{\jmath}^{M}\left(z_{0}\right) ; r, s\right)=N\left(f, H_{j}^{M} ; r, s\right)+S(f ; r) \quad(j=1, \cdots, q) .
$$

On the other hand, we have by Theorem 5.1, 


$$
(q-n-1) T(f ; r, s) \leq \sum_{j=1}^{q} N\left(f, H_{j}^{M}\left(z_{0}\right) ; r, s\right)+S(f ; r) .
$$

Hence we obtain

$$
(q-n-1) T(f ; r, s) \leq \sum_{j=1}^{q} N\left(f, H_{j}^{M} ; r, s\right)+S(f ; r) .
$$

Therefore we have the defect relation

$$
\sum_{j=1}^{q} \delta\left(f, H_{j}^{M I}\right) \leq n+1 .
$$

\section{§7. Further result}

In this section, we give a generalization of Theorem 4.4.

Before stating it, we show next lemmas.

Lemma 7.1. Let $g$ be a linearly non-degenerate holomorphic curve in $P^{m}(C)$ with a reduced representation $\left(g_{0}, \cdots, g_{m}\right)$. Assume that $N\left(g_{j} ; r, s\right)$ $=S\left(g_{k} / g_{l}\right)$ for any distinct $k$ and $l$. Then $g$ is non-degenerate over $\tilde{K}_{g}$.

Proof. Assume that $g_{0}, \cdots, g_{m}$ are linearly dependent over $\tilde{K}_{g}$. So there exist $a_{0}, \cdots, a_{m} \in \tilde{K}_{g}$ such that some $a_{j} \not \equiv 0$ and that $a_{0} g_{0}+\cdots+$ $a_{m} g_{m} \equiv 0$. Without loss of generality, we may assume that $a_{j} \not \equiv 0(0 \leq j$ $\leq k+1)$ and $a_{j} \equiv 0(k+2 \leq j \leq m)$, where $k+1 \leq m$, and that $g_{0}, \cdots$, $g_{k+1}$ are linearly independent over $\tilde{K}_{g}$. If $k=0$, we can immediately lead a contradiction. So, let $k \geq 1$.

Consider the holomorphic curve $h=\left(g_{0}: \cdots: g_{k}\right)$ in $P^{k}(C)$ and moving hyperplanes

$$
H_{j}^{M}(z)=\left\{w \in P^{k}(\boldsymbol{C}) \mid w_{j}=0\right\} \quad(0 \leq j \leq k)
$$

and $H_{k+1}^{M}$ with a representation $\left(a_{0}, \cdots, a_{k}\right)$ in $P^{k}(C)$. They are in general position and of lower order than $h$. By the assumption and the relation $a_{0} g_{0}+\cdots+a_{k} g_{k}=-a_{k+1} g_{k+1}$, we see that $\delta\left(g, H_{j}^{M}\right)=1(0 \leq j \leq k+1)$. This contradicts to Theorem 4.3. Hence we complete the proof of this lemma.

Q.E.D.

LEMMA 7.2. Let $f$ be a linearly non-degenerate holomorphic curve in $P^{n}(C)$ with a reduced representation $\tilde{f}=\left(f_{0}, \cdots, f_{n}\right)$ and let $g$ be a linearly non-degenerate holomorphic curve in $P^{m}(C)$ with a reduced representation $\tilde{g}=\left(g_{0}, \cdots, g_{m}\right)$. Assume that there are relations

$$
f_{j}=\sum_{k=0}^{m} a_{k}^{j} g_{k}, \quad a_{k}^{j} \in C(0 \leq j \leq n)
$$

and that for each $k=0, \cdots, m$, there is a $j(k)$ such that $a_{k}^{j(k)} \neq 0$. Moreover, if $N\left(g_{j} ; r, s\right)=S(g ; r)$ for $j=0, \cdots, m$, then 


$$
T(g ; r, s)=T(f ; r, s)+S(g ; r) .
$$

Proof. By (7.3), we have the inequality $\|\tilde{f}\| \leq C\|\tilde{g}\|$ for some $C>0$. Therefore we get

$$
T(f ; r, s) \leq T(g ; r, s)+O(1) .
$$

Now, we can choose $b_{0}, \cdots, b_{n} \in C$ such that $c_{k}:=\sum_{j=0}^{n} a_{k}^{j} b_{\jmath} \neq 0$. Consider hyperplanes

$$
H=\left\{w \in P^{n}(\boldsymbol{C}) \mid \sum_{j=0}^{n} b_{j} w_{j}=0\right\}
$$

in $P^{n}(C)$ and

$$
\begin{aligned}
L_{k} & =\left\{w \in P^{m}(C) \mid w_{k}=0\right\} \quad(0 \leq k \leq m), \\
L & =\left\{w \in P^{m}(C) \mid \sum_{k=0}^{m} c_{k} w_{k}=0\right\}
\end{aligned}
$$

in $P^{m}(C)$. Then by Theorem 5.1 , we have

$$
\begin{aligned}
T(g ; r, s) & \leq \sum_{k=0}^{m} N\left(g, L_{k} ; r, s\right)+N(g, L ; r, s)+S(g ; r) \\
& =N(g, L ; r, s)+S(g ; r) .
\end{aligned}
$$

Since $\sum_{k=0}^{m} c_{k} g_{k}=\sum_{j=0}^{n} b_{j} f_{j}$, we have

$$
N(g, L ; r, s)=N(f, H ; r, s) .
$$

Hence, we get by (3.5)

$$
\begin{aligned}
T(g ; r, s) & \leq N(f, H ; r, s)+S(g ; r) \\
& \leq T(f ; r, s)+S(g ; r) .
\end{aligned}
$$

Consequently, by (7.4), we obtain

$$
T(g ; r, s)=T(f ; r, s)+S(g ; r) .
$$

The generalization of Theorem 4.4 is the following:

THEOREM 7.5. Let $f$ be a linearly non-degenerate holomorphic curve in $P^{n}(\boldsymbol{C})$ with a reduced representation $\tilde{f}=\left(f_{0}, \cdots, f_{n}\right)$ given by $f_{j}=\sum_{k=1}^{m_{j}} f_{h}^{j}$, where $f_{1}^{j}, \cdots, f_{m_{j}}^{j}$ are entire functions which are linearly independent over $C(j=0, \cdots, n)$. Let $H_{j}^{M}$ be as in Theorem 4.4. Assume that $f$ is nondegenerate over $\tilde{K}_{f}$ and that $N\left(f_{k}^{j} ; r, s\right)=S\left(f_{k}^{j} / f_{m}^{l} ; r\right)$ if $f_{k}^{j} / f_{m}^{l}$ is not constant. Then

$$
\sum_{j=1}^{q} \delta\left(f, H_{j}^{M}\right) \leq n+1 .
$$

Proof. Choose $g_{0}, \cdots, g_{m}$ from $f_{k}^{j}\left(1 \leq k \leq m_{j}, 0 \leq j \leq n\right)$ such that 
$\left\{g_{0}, \cdots, g_{m}\right\}$ is a base of the vector space over $C$ spanned by $f_{k}^{j}(1 \leq k \leq$ $\left.m_{j}, 0 \leq j \leq n\right)$. Let $g$ be a holomorphic curve in $P^{n}(\boldsymbol{C})$ with a reduced representation $\tilde{g}=\left(g_{0} / h, \cdots, g_{m} / h\right)$, where $h$ is an entire function such that $g_{0} / h, \cdots, g_{m} / h$ are entire functions without common zero. By Lemma 7.1, $g$ is non-degenerate over $\tilde{K}_{g}$. It is easy to check that $\tilde{K}_{f} \subset \tilde{K}_{g}$.

We define entire functions $b_{k}^{j}(1 \leq j \leq q, 0 \leq k \leq m)$ by the equations

$$
a_{0}^{j} f_{0}+\cdots+a_{n}^{j} f_{n}=b_{0}^{j} g_{0}+\cdots+b_{m}^{j} g_{m} \not \equiv 0 \quad(1 \leq j \leq q) .
$$

Since $b_{k}^{j}$ are linear combinations of $a_{0}^{j}, \cdots, a_{n}^{j}$ with complex coefficients, we see that $b_{l}^{j} / b_{k}^{j} \in \tilde{K}_{g}$ if $b_{k}^{j} \not \equiv 0$. Let $d_{j}$ be a common factor of $a_{0}^{j}, \cdots, a_{m}^{j}$ and let $L_{j}^{M}$ be a moving hyperplane in $P^{m}(C)$ with a reduced representation $b_{j}=\left(b_{0}^{j} / d_{j}, \cdots, b_{m}^{j} / d_{j}\right)$. Set $a_{j}=\left(a_{0}^{j}, \cdots, a_{n}^{j}\right)$. Then $\left(\tilde{f}, a_{j}\right)=h d_{j}\left(\tilde{g}, b_{j}\right)$. Hence we have

$$
N\left(f, H_{j}^{M} ; r, s\right)=N\left(g, L_{j}^{M} ; r, s\right)+N\left(h d_{j} ; r, s\right) .
$$

We choose $z_{0}$ of $C$ such that $b_{k}^{j}\left(z_{0}\right) \neq 0$ if $b_{k}^{j} \not \equiv 0$ and $H_{1}^{M}\left(z_{0}\right), \cdots, H_{q}^{M}\left(z_{0}\right)$ are in general position. Then by Lemma 6.1, we get

$$
N\left(g, L_{j}^{M}\left(z_{0}\right) ; r, s\right)=N\left(g, L_{j}^{M} ; r, s\right)+S(g ; r) .
$$

Furthermore we have

$$
N\left(g, L_{j}^{M}\left(z_{0}\right) ; r, s\right)+N(h ; r, s)=N\left(f, H_{j}^{M}\left(z_{0}\right) ; r, s\right)
$$

by $\left(\tilde{f}, a_{j}\left(z_{0}\right)\right)=h d_{j}\left(z_{0}\right)\left(\tilde{g}, b_{j}\left(z_{0}\right)\right)$. Since $N\left(d_{j} ; r, s\right)=o(T(f ; r, s))$ by Proposition $3.8, N(h ; r, s)$ is $S(f ; r)$ and $S(g ; r)$ is $S(f ; r)$ by Lemma 7.2 , we obtain

$$
N\left(f, H_{j}^{M}\left(z_{0}\right) ; r, s\right)=N\left(f, H_{j}^{M} ; r, s\right)+S(f ; r)
$$

by (7.6), (7.7) and (7.8). Hence using Theorem 5.1, we have

$$
\begin{aligned}
(q-n-1) T(f ; r, s) & \leq \sum_{j=1}^{q} N\left(f, H_{j}^{M}\left(z_{0}\right) ; r, s\right)+S(f ; r) \\
& \leq \sum_{j=1}^{q} N\left(f, H_{j}^{M} ; r, s\right)+S(f ; r) .
\end{aligned}
$$

Therefore we obtain the defect relation

$$
\sum_{j=1}^{q} \delta\left(f, H_{j}^{M}\right) \leq n+1 .
$$

The most typical case of Theorem 4.4 is that $f_{j}=\exp h_{j}$, where $h_{j}$ are entire functions, and $\alpha_{k}^{j}$ are polynomials. 


\section{REFERENCES}

[1] P. Griffiths and J. King, Nevanlinna theory and holomorphic mappings between algebraic varieties, Acta Math., 130 (1973), 145-220.

[2] S. Mori, Remarks on holomorphic mappings, Contemporary Math. Vol. 25, 1983, 101-113.

[ 3 ] B. V. Shabat, Distribution of values of holomorphic mappings, Amer. Math. Soc., Providence, R.I., 1985.

[4] W. Stoll, An extension of the theorem of Steinmetz-Nevanlinna to holomorphic curves, Math. Ann., 282 (1988), 185-222.

[5] - Value distribution theory for meromorphic maps, Aspects of Mathematics, E7, Vieweg, 1985.

Department of Mathematics

Faculty of Science

Kanazawa University

Marunouchi, Kanazawa 920

Japan 